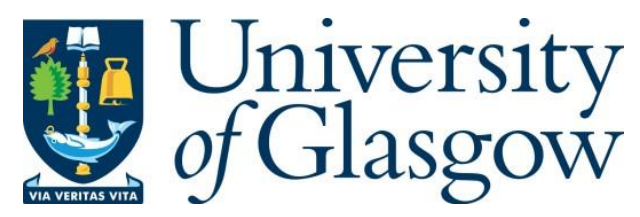

Sattar, N., Gill, J. M.R. and Alazawi, W. (2020) Improving prevention strategies for cardiometabolic disease. Nature Medicine, 26(3), pp. 320-325.

There may be differences between this version and the published version. You are advised to consult the publisher's version if you wish to cite from it.

$\underline{\text { http://eprints.gla.ac.uk/212691/ }}$

Deposited on: 3 April 2020

Enlighten - Research publications by members of the University of Glasgow http://eprints.gla.ac.uk 


\title{
Unifying prevention strategies for cardiometabolic disease
}

\author{
Sattar $\mathrm{N}^{* 1}$, Gill JMR ${ }^{1}$, Alazawi $\mathrm{W}^{2}$ \\ ${ }^{1}$ University of Glasgow, \\ ${ }^{2}$ Queen Mary University of London
}

\section{Corresponding Author}

Naveed Sattar

University of Glasgow

Institute of Cardiovascular \& Medical Sciences

BHF Glasgow Cardiovascular Research Centre

126 University Place

Glasgow

G12 8TA

UK

ORCID: 0000-0002-1604-2593

Email: naveed.sattar@glasgow.ac.uk

Tel: +44(0)1413303419

Word count: 3417

2 boxes; 2 original figures; 48 references 
This is a growing burden of cardiometabolic disease in many parts of the world. Despite some progress in their prevention, more can be done to tackle risks of their development in the community and in different speciality clinics. Currently, the identification and management of those at elevated risk of developing cardiovascular disease, diabetes, or with conditions such fatty liver disease remains fragmented, and is not linked to constructive lifestyle advice. In this Perspective, we argue for a more consistent weight management approach, alongside a holistic assessment of risk for developing cardiometabolic diseases, offering patients a range of simple or more intensive evidence-based options in an empathetic manner with encouragement for repeated attempts and a willingness to embrace failure. 


\section{Introduction}

Over the last 3-4 decades, considerable progress in the prevention and treatment of cardiovascular diseases (CVD) has been made. The major causal risk factors of these diseases are smoking, high cholesterol and high blood pressure and the rates or incidence of these has declined over the last several decades ${ }^{1}$ in most high-income countries. The result has been a remarkable decline in cardiovascular disease-induced deaths such that more people in high income countries now die from cancer than cardiovascular disease ${ }^{2}$. However, at the same time obesity rates have escalated markedly over the last few decades ${ }^{3}$ as has the prevalence of type 2 diabetes $^{4}$. This increase in type 2 diabetes is linked variably in different parts of the world to rising rates of obesity, aging populations as well as more testing for glycaemia leading to fewer undiagnosed cases.

This changing pattern of disease presents substantial challenges to health care; it should serve as a call to improve prevention of cardiovascular disease by better targeting those at risk. At the same time, there is a need to improve advice on lifestyle changes in clinical care such that the medical profession offers individuals a range of simple evidenced-based options towards sustainable lifestyle changes. Such lifestyle changes can lessen risks for several cardiometabolic diseases, including cardiovascular diseases, diabetes and non-alcoholic fatty liver disease, plus potentially some cancers, as well as improve quality of life. Of course, public health strategies can have major benefits in reducing populations' risks for cardiometabolic diseases by, for example, taxing sugar-rich drinks or related measures but such issues are beyond the scope of this article. We present below a summary of the medical challenges towards more holistic testing for risks and in introducing lifestyle interventions as well as a list of potential options to help address these.

\section{Trends in cardiovascular and diabetes prevalence $\mid$}

It is now not disputed that smoking, high blood pressure and high blood lipid levels (cholesterol more strongly than triglyceride) are causal risk factors for CVD. Multiple guidelines around the world now place great emphasis on measuring these risk factors in people when they attend for care in either 
primary practice or their secondary health care providers ${ }^{5}$. However, it remains debated at what age apparently healthy people are best screened and how to carry out this screening efficiently with respect to time and resources. Some recent advances in risk screening have helped to simplify the process (Box 1). There remains a need, however, to better identify individuals at higher risk of developing CVD and metabolic complications. Furthermore, current intervention strategies are not impacting the rising obesity rates and its associated effects on disease risk and quality of life. The recent trend in high income countries of reducing CVD death rates may soon be reversed by societal adiposity shifts, as obesity is causally linked to many cardiovascular diseases. ${ }^{6}$ To identify those at risk requires more opportunities for individuals to be tested along with providing individuals with better education on the risk factors for CVD and diabetes and how to mitigate them.

In high-income countries, robust advances have also been made in diabetes management; CVD hospitalisations and CVD death rates in those with diabetes have come down over the last few decades broadly commensurate with trends in the general population, though more can be done to lower the associated risks of disease in diabetes. ${ }^{7}$ Concerns going forwards include more younger people developing type 2 diabetes; in high income countries, such individuals are more often severely obese and their glucose levels rise faster over time compared with those who develop diabetes much later in life. ${ }^{8,9}$ Furthermore, the rising number of people with type 2 diabetes in general is stretching health services; part of this is due to better survival of people with diabetes in high income countries. This pattern is also extending comorbidity levels in diabetes, as less premature deaths due to CVD causes means patients have more time to develop other diabetes complications.$^{10}$

There is, however, good news with better management strategies at different stages of diabetes. Recent trials have revealed classes of diabetes drugs such as SGLT2 inhibitors, drugs which enhance urinary excretion of sugar, salt and water, and GLP-1RA, drugs which help improve the release of insulin after meals and aid weight loss, that lessen a range of hard cardiovascular and cardiorenal outcomes in those with diabetes and existing cardiovascular diseases. ${ }^{11,12}$. As these new classes of drugs are considerably more expensive than some of the more established anti-hyperglycaemia 
medicines, the challenge is how to optimise their use in a cost-effective manner. At the same time, there has been progress in diabetes prevention. Early examination of the English diabetes prevention programme shows good weight loss in individuals adhering to the programme but improvements in program engagement, retention and completion are required ${ }^{13}$. Currently, the focus of these programmes is to identify those at highest risk and introduce lifestyle changes to slow or halt progression to diabetes. However, as the pre-diabetes population has on average a higher prevalence of hypertension, obesity and dyslipidaemia, those identified at high risk of diabetes would also benefit from formal assessment of their $\mathrm{CV}$ risk ${ }^{14}$. Thus, going forwards, there is merit in discussing how best to screen relevant people for both CVD and diabetes risks. ${ }^{15}$ Official guidance would help target blood tests to those at higher risk.

\section{Developing effective screening}

Screening for cardiometabolic conditions and their associated risks is often done initially by trained allied health professionals in primary care to save on time and costs. Nurses or other trained staff can collect routine weight, height and blood pressure and ask some simple questions on smoking, alcohol intake and family history of CVD and T2DM. All the collected information could be entered into one combined risk scoring system that simultaneously reads out separate non-laboratory-based risk estimates for both conditions. This is possible since several risk parameters such as age, sex, BMI, blood pressure, and socioeconomic status predict both conditions, even if their magnitudes of association differ, in some cases markedly (e.g. higher BMI is much more strongly linked to risk for diabetes than to risk for $\mathrm{CVD}^{16}$ ). Such a risk score could help inform which people warrant further formal blood tests to analyse in more detail their risk. For those at risk of CVD: blood test for nonfasting lipids, liver biochemistry and kidney function tests would be actioned. For those at risk of diabetes: glycated haemoglobin [HbA1c], a test of average sugar level over the last two to three months, or fasting glucose if patients fasted, are easy additions. For simplicity, doctors might wish to run all tests to be comprehensive (Figure 1), though laboratory costs and workload of conducting such tests are not insignificant. 


\section{The potential advantages and challenges to screening}

Initial risk scoring is not difficult and with education could be done relatively quickly by allied health professionals. It could also be done in a range of non-clinical settings where people often attend to make initial checks more accessible. A recent example is the community based screening for diabetes in barbershops to identify black men with undiagnosed diabetes ${ }^{17}$. Since many people tested will be at low absolute risk for both CVD and diabetes, only a minority at higher or intermediate risk (or those with a family history of premature cardiovascular disease) would require formal blood testing. From this latter group, a further subgroup would warrant potential therapy or specific advice to be delivered by doctors. As age is a major risk factor for both conditions, blood tests would be more commonly done in those in middle and older age, and some guidelines advocate risk screening or CVD above 40 or 50 years of age. In the younger groups, those found to be at lower risk could be rescreened in 3-5 years, with perhaps more frequent rescreening in older groups.

There are of course multiple challenges to widening health checks as has been seen with the English Vascular Health checks programme that has low coverage and poor statin prescription in high risk individuals ${ }^{18}$. Notably, fewer people from socioeconomically deprived or black and minority ethnic communities attend these checks in the UK. Thus, widening access to health checks in pharmacies and other local places (e.g. community centres, places of worship, barbershops etc.) in the UK or elsewhere could help as could a well thought out, empathic public communication programmes to highlight the benefits of a simple and quick risk assessment and of simple lifestyle advice. Whilst prior trials have not shown outcome benefits from nationwide screening programmes, at least over 10 years ${ }^{19}$ (longer follow-up may be needed), the evidence base on how to help people make lifestyle improvements has improved and the cost-effectiveness of some preventative (lipid-lowering, antihypertensive) treatments has also increased as drug costs have declined ${ }^{20,21}$. The medical profession is also slowly improving its communication of risk so that people can better understand the pros and cons of any prevention approach, but more can be done. The aim should therefore be to keep 
improving and refining preventative efforts towards individual behaviour change as well as societal policies that address risk factors such as smoking and diet.

\section{Where should screening happen}

Doctors are increasingly familiar with a range of conditions linked to greater CV risk (such as hypertension, rheumatoid arthritis, systemic lupus erythematosus, erectile dysfunction, severe mental illness $^{22}$ ) as well as those linked to diabetes risk (such as obesity, fatty liver, psoriasis, hypertension, gestational diabetes). Given the ease of adding non-fasting lipids, biochemistry and/or HbA1c (glyeated haemoglobin, a test that captures average blood glueose levels over a few months) in all clinics then screening for CVD, diabetes and, where relevant, other outcomes such liver risk should not be an onerous process for clinicians in any speciality. Electronic records in many high income countries are now able to link hospitals to primary care records so it should be easy to check whether patients have had recent such tests in primary care; but if not, there is no reason why these tests cannot be added in those with a range of existing conditions known to be associated with higher cardiometabolic risks to give a more holistic assessment of a patient's risk for cardiovascular disease and diabetes (Figure 1). Whether in the future, a unified risk scoring system that simultaneously calculates risks for subcategories of cardiovascular disease and other diseases rising in prevalence such as heart failure, is helpful requires investigation. In reality, in the era of rising comorbidity, we need more comprehensive risk scoring systems to enhance the push towards holistic medicine leading to tailored advice or choice of therapies as well as more cross-speciality training to improve the comprehensiveness of medical care. Notably, experts have already proposed setting up a new class of cardiometabolic specialists that have expertise across traditional boundaries ${ }^{23}$.

\section{Combining screening with lifestyle advice}

There have been widespread improvements in treating diseases such as cancers, cardiac and autoimmune disease once they are diagnosed or in recognising and treating risk factors such as blood pressure, cholesterol or glucose when they are significantly elevated. However, improvement in prevention (or even reversal) of disease via lifestyle changes has been lagging behind. Few individuals get formal assessment of their adiposity levels or ever asked about activity levels ${ }^{24}$, and 
even fewer receive any sensible advice on how to address either. Furthermore, few doctors have any training in these areas ${ }^{25}$ and there is evidence that while patients think and say that they follow dietary advice, most do not. If truth be told, few doctors currently offer any dietary advice fearing to do so will take too much time and that most patients will fail to lose weight or will easily put it back on. This pattern needs to change if lifestyle is a major contributor to many chronic diseases including but not limited to diabetes, CVD, fatty liver disease, multiple cancers, renal disease, heart failure, some skin diseases like gout and psoriasis, as well as osteoarthritis and several lung conditions. What is less well understood is that whilst weight gain rapidly increases diabetes risks due to almost immediate increases in ectopic fat depots it may take longer, perhaps decades, for small to modest increases in weight to translate into cardiovascular outcomes, as adiposity takes time to accelerate atherosclerosis. The same may be true for other chronic diseases.

Nevertheless, it is important to note that if better lifestyle advice helps some people improve their future weight trajectory (by slowing or ceasing weight gain or losing weight), this could have considerable benefits over the longer term by delaying or preventing a range of outcomes. In the reverse direction, weight loss can almost immediately lead to $\mathrm{HbAlc}$ reduction or indeed diabetes reversal $^{26}$, whereas it may take years for small weight or lifestyle changes to show benefits on CVD outcomes $^{27}$. This is one reason why any potential impact of obesity per se on population trends in CVD or other outcomes usually take time to emerge. That noted, we must not lose sight of the impact of even modest weight changes on quality of life, mental health and self-esteem; the health care profession is waking up to the importance of such "softer" patient reported outcomes, and nearly all overweight or obese patients are genuinely happy to discuss their weight ${ }^{28}$ and would wish to lose weight.

Fortunately, there has been increase in recent understanding as to how to help people make sustainable lifestyle changes, particularly diet. We would argue that such new knowledge needs to be rapidly coalesced into something easily communicated to patients so that we start to give specific, actionable, evidenced-based advice that is consistent and easily digestible (Figure 2). It is also clear 
we must do so in an empathetic manner so that patients understand we are there to help them and not to chastise or engage in any form of "fat-shaming". We believe the evidence now allows a range of options to be offered to patients from simple dietary changes to more intensive options. We also believe that such advice can be collated into a simple leaflet, an extension of the top ten tips concept ${ }^{29}$, directing patients to further evidence-based option. This range of options should include the potential for some to be referred to, or join a weight loss $\operatorname{clinic}^{30}$ as well as the option of low calorie diet ${ }^{31,32}$ for some or drugs for others to help aid or kick start weight loss. Of course, a minority of individuals may benefit from and progress to bariatric surgery or the emerging endobariatric techniques.

\section{Studying the approach}

Lifestyle trials are much harder than drug trials and this is why we have less robust evidence. The result is less emphasis on lifestyle in medical guidelines, whereas, if we could help many more make sustainable improvements in their lifestyle, the impact might be greater than we imagine. Recent trials showing the robust benefits of referring willing patients to commercial weight loss providers ${ }^{33}$, as well as low calorie diets (with initial total dietary replacement) in type 2 diabetes $^{31}$, non-diabetes ${ }^{32}$, and psoriasis ${ }^{34}$, have provided new opportunities to test the impact of lifestyle on other diseases. There are ongoing trials of this approach in patients with atrial fibrillation ${ }^{35}$ and other areas such as liver disease. In a few years' time, one might see trial evidence testing the potential benefits of more significant and sustainable weight loss in a range of other conditions (e.g. osteoarthritis, NASH, heart failure with preserved ejection fraction, sleep apnoea, resistant hypertension, post gestational diabetes) for which differential evidence levels exist for the benefits of weight loss. At the same time, more work is needed to better help people maintain weight loss after the initial period of dietary change. As the evidence base is improving, on the background of ever rising obesity, more research funds should be and appear to be, directed into prevention of disease than in treating it when it occurs. As noted before, the medical profession cannot directly alter the obesogenic environment, but we can offer a range of evidence-based lifestyle advice to our patients on how to lose weight sustainably or improve their weight trajectory. 


\section{Communicating advice}

There is clear evidence that increasing physical activity is associated with positive health outcomes, with any increase in activity being beneficial and more being better ${ }^{36}$. Importantly, building physical activity into the day, using approaches such as active $\operatorname{trave}^{37}$ or by incorporating multiple small amounts activity throughout the day can provide tangible benefits ${ }^{38}$. Pedometer-supported behaviour change approaches which incorporate setting of progressive physical activity goals, and provide selfmonitoring and feedback of activity levels are effective at increasing physical activity levels ${ }^{39}$, and there is emerging evidence that smartphone-based approaches can be effective for promoting physical activity ${ }^{40}$. However, it is important that targets are personalised and realistic - for example, for someone who is very inactive, initially increasing step count by 1000 per day, equivalent to about 10 extra minutes of walking, can potentially provide benefit and provides a platform for further increases in activity with time. The amount of physical activity needed to substantially lower risk of adverse health outcomes may be relatively small. Data from a recent meta-analysis of the association between accelerometer-measured (rather than self-reported) physical activity in eight studies with over 240,000 person-years of follow-up suggests that undertaking just 6 minutes per day of moderate-tovigorous physical activity was associated with a $\sim 30 \%$ lower risk of mortality in maximally-adjusted models ${ }^{41}$, and in a randomised trial with $>1100$ participants aiming to increase physical activity and reduce sedentary time in overweight male soccer fans, we found that the intervention increased accelerometer-measured physical activity by $\sim 6$ minutes per day at 12 months, but this was sufficient to induce positive changes in biomarkers of cardiometabolic risk, including reducing insulin resistance by $15 \%{ }^{42}$.

Historically, there has been an emphasis on aerobic physical activities, typified by walking, in physical activity messaging, but there is now also increasing evidence that muscular strength and engaging in muscle strengthening exercise is likely to contribute to health-related outcomes. Data from the PURE study and UK Biobank show that low grip strength is strongly associated with risk of mortality (16-20\% higher risk per $5 \mathrm{~kg}$ lower strength) and incidence of several chronic diseases ${ }^{43,44}$, and engaging in muscle-strengthening exercises appears to be associated with benefits over and above 
aerobic activity for risk of mortality ${ }^{45,46}$. This is reflected in the recent UK guidelines on physical activity ${ }^{47}$ which have emphasised the importance of building and maintaining strength. We need to do more to equip healthcare professionals to deliver simple evidence-based interventions which include both muscular strength and aerobic activity at levels which are eminently more achievable than many people perceive.

\section{Conclusions}

With reductions in CVD death rates over the last few decades, but simultaneous rises in obesity and related conditions, metabolic disease is on the rise worldwide and beginning to be seen earlier in life. Obesity is also on the rise and a driver of comorbidity. Thus, it is time the medical profession develops better management protocols to help tackle this issue which has major impacts on patients' quality of life and future risks of disease - such work could in turn improve how lifestyle advice is delivered to the public. The important message is the need for the medical profession to develop a more empathetic and helpful approach to obesity so that patients feel encouraged to try to make changes in their lifestyle and better understand how to make these. Simple, specific, actionable, evidence-based advice is needed as well as an atmosphere that encourages people to try different options that have supporting evidence without the fear of being chastised if they do not succeed. At the same time, a parallel focus on preventing development of obesity in those at risk is needed, as well as more trials of meaningful weight loss as a treatment in a range of conditions. In addition, simple ways to assess CVD risk can be widened so all specialities can do this easily. There is no need for overnight fasting for blood testing and most people can accurately complete a simple risk score so all specialities can screen for CVD risk or indeed diabetes. Such screening can also be done or commenced in many locations outside of hospitals thereby encouraging more at risk and often harder to reach to be screened. We also need better tools to explain risks to the public in a way that incentivises them to engage with lifestyle better and, where relevant, to be understand the benefits of being more compliant with their preventative medications. In short, it is time to up our game on prevention with a more empathetic and helpful focus on lifestyle. 


\section{Figure legends}

Figure 1. Risk assessment for cardiometabolic disease.

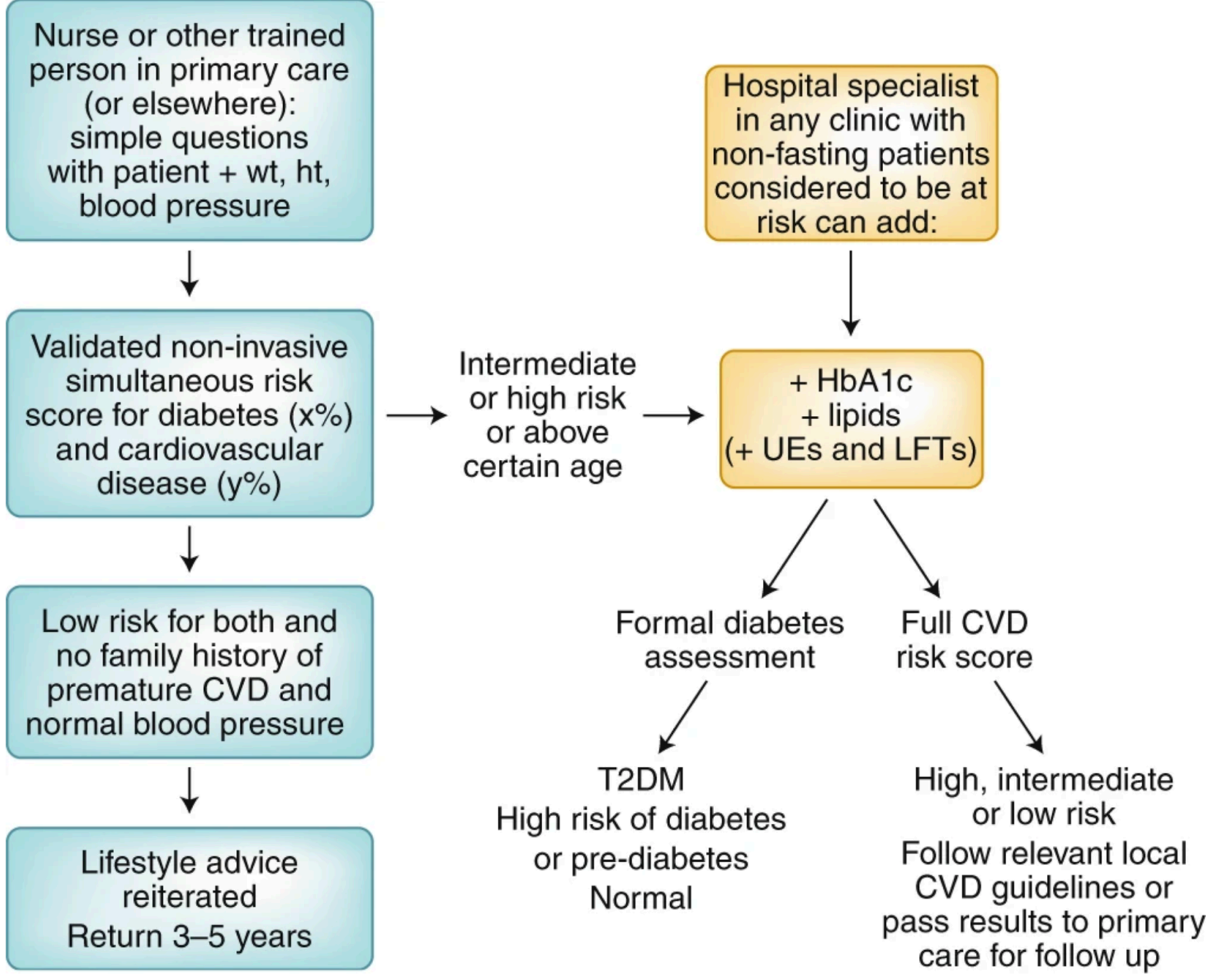

Risk scoring for CVD or diabetes could take place in a two-stage process. The first step would be non-laboratory based, requiring only blood pressure, weight and height along with specific questions. In those above a certain age, or shown to be at elevated risk for either or both of CVD and diabetes, then progression to measurement of lipids and $\mathrm{HbA} 1 \mathrm{c}$ (or fasting glucose if appropriate) can be done easily in nearly all clinical settings. The key concepts are that the simultaneous assessment of risks for a range of condition is now possible but that further work is needed to determine how best to do this. Determining risks for several conditions may better motivate people to improve their lifestyles or, as recommended, comply with preventative medicines. 
Figure 2. Simple, specific, actionable, evidence-based advice for individuals on lifestyle.

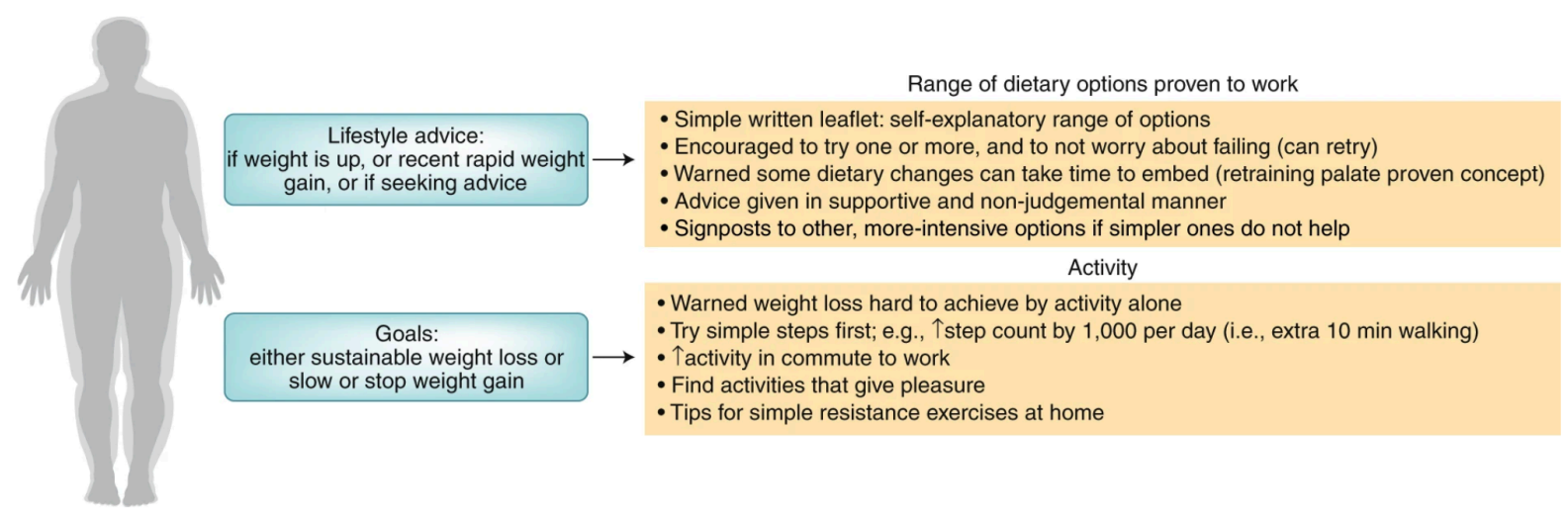

The evidence base for approaches that result in diet improvement and activity increase in individuals has improved substantially in the last decade such that it may now be possible to give patients much simpler advice in a short leaflet of options (and extension of the 10 tips concept ${ }^{29}$ ) that is specific and more easily actioned. By moving in this direction, we believe it is possible to substantially improve the routine "prescription" of lifestyle advice in all medical settings. This should lead, in turn, to more patients understanding how to achieve beneficial changes and adopting some, although clearly some individuals will require and only benefit from more intensive options. We also suggest a need for all such advice to be given in a much supportive and non-judgemental manner. 
Box 1

Options for Screening for cardiovascular disease, diabetes and liver disease:

- Multiple risk scores for cardiovascular disease based on simple questions, physical measures and simple blood tests are now available online e.g. JBS3 in the UK

[http://www.jbs3risk.com/] or the joint AHA/ACC score in the USA

[ http://www.cvriskcalculator.com/]. Risk scoring need not be difficult, and can be repeated easily over time.

- Measurements of circulating lipid levels which are needed for full cardiovascular risk scores, can be measured in the non-fasting state for CV risk factor scores and total and HDL-

cholesterol suffice for most purposes. In low-income countries where blood tests are harder to obtain, the addition of body mass index ${ }^{48}$ and grip strength ${ }^{44}$ may aid risk scores.

- Online diabetes risks are plentiful e.g. https://qdiabetes.org/ or http://riskscore.diabetes.org.uk/. These can be done very quickly and help guide which people require formal blood testing for $\mathrm{HbAlc}$ or fasting glucose.

- Multiple risk scores for liver fibrosis are now available online. Patients with low risk scores can be safely monitored in non-specialist setting. Patients with indeterminate and high-risk scores should be referred for further testing 
Box 2

Future questions/challenges with respect to cardio-metabolic risk screening

- Is it possible and desirable to develop a risk algorithm which simultaneously reads out 10year and life time risks for diabetes, cardiovascular disease and its subcomponents and risk of mortality? Could such a risk algorithm usefully add risk for conditions such as heart failure or other conditions?

- Can the presentation of all risk algorithms be improved so that people are better able to understand their risks, for example how they compare with others of their age, and how to mitigate them?

- Would better explanations of risks motivate more people to adopt lifestyle changes that lessen risks and, if relevant, commence and adhere to preventative medications?

- Can non-invasive risk assessments incorporating blood pressure and weight and height be safely expanded to pharmacies, work places, sports centres or other community settings to provide greater options to have an initial risk score for CVD outcomes and diabetes and to receive appropriate advice on lifestyle changes or to attend their primary care provider for further assessment? 


\section{References}

1. Sun, X. \& Du, T. Trends in cardiovascular risk factors among U.S. men and women with and without diabetes, 1988-2014. BMC Public Health 17, 893 (2017).

2. Dagenais, G. R. et al. Variations in common diseases, hospital admissions, and deaths in middle-aged adults in 21 countries from five continents (PURE): a prospective cohort study. Lancet (2019). doi:10.1016/s0140-6736(19)32007-0

3. Blüher, M. Obesity: global epidemiology and pathogenesis. Nature Reviews Endocrinology 15, 288-298 (2019).

4. Danaei, G. et al. National, regional, and global trends in fasting plasma glucose and diabetes prevalence since 1980: Systematic analysis of health examination surveys and epidemiological studies with 370 country-years and $2 \cdot 7$ million participants. Lancet 378, 31-40 (2011).

5. Piepoli, M. F. et al. 2016 European Guidelines on cardiovascular disease prevention in clinical practice: The Sixth Joint Task Force of the European Society of Cardiology and Other Societies on Cardiovascular Disease Prevention in Clinical Practice. Eur. Heart J. 37, 2315$2381(2016)$.

6. Larsson, S. C., Bäck, M., Rees, J. M. B., Mason, A. M. \& Burgess, S. Body mass index and body composition in relation to 14 cardiovascular conditions in UK Biobank: a Mendelian randomization study. Eur. Heart J. (2019). doi:10.1093/eurheartj/ehz388

7. Gregg, E. W., Sattar, N. \& Ali, M. K. The changing face of diabetes complications. The Lancet Diabetes and Endocrinology 4, 537-547 (2016).

8. Steinarsson, A. O. et al. Short-term progression of cardiometabolic risk factors in relation to age at type 2 diabetes diagnosis: a longitudinal observational study of 100,606 individuals from the Swedish National Diabetes Register. Diabetologia 61, 599-606 (2018).

9. Donnelly, L. A. et al. Rates of glycaemic deterioration in a real-world population with type 2 diabetes. Diabetologia 61, 607-615 (2018).

10. Hussain, S. \& Chowdhury, T. A. The Impact of Comorbidities on the Pharmacological Management of Type 2 Diabetes Mellitus. Drugs 79, 231-242 (2019). 
11. Zelniker, T. A. et al. SGLT2 inhibitors for primary and secondary prevention of cardiovascular and renal outcomes in type 2 diabetes: a systematic review and meta-analysis of cardiovascular outcome trials. Lancet 393, 31-39 (2019).

12. Kristensen, S. L. et al. Cardiovascular, mortality, and kidney outcomes with GLP-1 receptor agonists in patients with type 2 diabetes: a systematic review and meta-analysis of cardiovascular outcome trials. Lancet Diabetes Endocrinol. 7, 776-785 (2019).

13. Valabhji, J. et al. Early Outcomes From the English National Health Service Diabetes Prevention Program. Diabetes Care (2019). doi:10.2337/dc19-1425

14. Welsh, C. et al. Glycated Hemoglobin, Prediabetes, and the Links to Cardiovascular Disease: Data From UK Biobank. Diabetes Care (2019). doi:10.2337/dc19-1683

15. Preiss, D., Khunti, K. \& Sattar, N. Combined cardiovascular and diabetes risk assessment in primary care. Diabet. Med. 28, 19-22 (2011).

16. Sattar, N. et al. Can metabolic syndrome usefully predict cardiovascular disease and diabetes? Outcome data from two prospective studies. Lancet (London, England) 371, 1927-35 (2008).

17. Osorio, M. et al. Community-Based Hemoglobin $\mathrm{A}_{1 \mathrm{C}}$ Testing in Barbershops to Identify Black Men With Undiagnosed Diabetes. JAMA Intern. Med. (2020). doi:10.1001/jamainternmed.2019.6867

18. Chang, K. C.-M. et al. Coverage of a national cardiovascular risk assessment and management programme (NHS Health Check): Retrospective database study. Prev. Med. (Baltim). 78, 1-8 (2015).

19. Jørgensen, T. et al. Effect of screening and lifestyle counselling on incidence of ischaemic heart disease in general population: Inter99 randomised trial. $B M J \mathbf{3 4 8},(2014)$.

20. Cholesterol Treatment Trialists' (CTT) Collaborators et al. The effects of lowering LDL cholesterol with statin therapy in people at low risk of vascular disease: meta-analysis of individual data from 27 randomised trials. Lancet (London, England) 380, 581-590 (2012).

21. Williams, B. et al. 2018 ESC/ESH Guidelines for the management of arterial hypertension. European Heart Journal 39, 3021-3104 (2018).

22. Hippisley-Cox, J., Coupland, C. \& Brindle, P. Development and validation of QRISK3 risk 
prediction algorithms to estimate future risk of cardiovascular disease: Prospective cohort study. BMJ 357, j2099 (2017).

23. Chang, L.-S., Vaduganathan, M., Plutzky, J. \& Aroda, V. R. Bridging the Gap for Patients with Diabetes and Cardiovascular Disease Through Cardiometabolic Collaboration. Curr. Diab. Rep. 19, 157 (2019).

24. Nicholson, B. D. et al. Determinants and extent of weight recording in UK primary care: an analysis of 5 million adults' electronic health records from 2000 to 2017. BMC Med. 17, 222 (2019).

25. Barnard, N. D. Ignorance of Nutrition Is No Longer Defensible. JAMA Intern. Med. 179, 1021 (2019).

26. Lim, E. L. et al. Reversal of type 2 diabetes: normalisation of beta cell function in association with decreased pancreas and liver triacylglycerol. Diabetologia 54, 2506-14 (2011).

27. Gong, Q. et al. Morbidity and mortality after lifestyle intervention for people with impaired glucose tolerance: 30-year results of the Da Qing Diabetes Prevention Outcome Study. Lancet Diabetes Endocrinol. 7, 452-461 (2019).

28. Aveyard, P. et al. Screening and brief intervention for obesity in primary care: a parallel, twoarm, randomised trial. Lancet (London, England) 388, 2492-2500 (2016).

29. Kliemann, N., Croker, H., Johnson, F. \& Beeken, R. J. Development of the Top Tips HabitBased Weight Loss App and Preliminary Indications of Its Usage, Effectiveness, and Acceptability: Mixed-Methods Pilot Study. JMIR mHealth uHealth 7, e12326 (2019).

30. Jebb, S. A. et al. Primary care referral to a commercial provider for weight loss treatment versus standard care: a randomised controlled trial. Lancet (London, England) 378, 1485-92 (2011).

31. Lean, M. E. J. et al. Durability of a primary care-led weight-management intervention for remission of type 2 diabetes: 2-year results of the DiRECT open-label, cluster-randomised trial. Lancet Diabetes Endocrinol. 7, 344-355 (2019).

32. Astbury, N. M. et al. Doctor Referral of Overweight People to Low Energy total diet replacement Treatment (DROPLET): pragmatic randomised controlled trial. BMJ 362, k3760 
(2018).

33. Ahern, A. L. et al. Extended and standard duration weight-loss programme referrals for adults in primary care (WRAP): a randomised controlled trial. Lancet (London, England) 389, 2214 2225 (2017).

34. Jensen, P. et al. Long-term effects of weight reduction on the severity of psoriasis in a cohort derived from a randomized trial: a prospective observational follow-up study. Am. J. Clin. Nutr. 104, 259-65 (2016).

35. LOSE-AF: Can Weight Loss Help Patients With Atrial Fibrillation? Available at: https://clinicaltrials.gov/ct2/show/NCT03713775. (Accessed: 23rd September 2019)

36. Piercy, K. L. et al. The Physical Activity Guidelines for Americans. JAMA 320, 2020 (2018).

37. Celis-Morales, C. A. et al. Association between active commuting and incident cardiovascular disease, cancer, and mortality: prospective cohort study. BMJ 357, j1456 (2017).

38. Murphy, M. H., Lahart, I., Carlin, A. \& Murtagh, E. The Effects of Continuous Compared to Accumulated Exercise on Health: A Meta-Analytic Review. Sports Med. 49, 1585-1607 (2019).

39. Harris, T. et al. Effect of a Primary Care Walking Intervention with and without Nurse Support on Physical Activity Levels in 45- to 75-Year-Olds: The Pedometer And Consultation Evaluation (PACE-UP) Cluster Randomised Clinical Trial. PLoS Med. 14, e1002210 (2017).

40. Feter, N., Dos Santos, T. S., Caputo, E. L. \& da Silva, M. C. What is the role of smartphones on physical activity promotion? A systematic review and meta-analysis. Int. J. Public Health 64, 679-690 (2019).

41. Ekelund, U. et al. Dose-response associations between accelerometry measured physical activity and sedentary time and all cause mortality: systematic review and harmonised metaanalysis. BMJ 366, 14570 (2019).

42. Wyke, S. et al. The effect of a programme to improve men's sedentary time and physical activity: The European Fans in Training (EuroFIT) randomised controlled trial. PLoS Med. 16, e1002736 (2019).

43. Leong, D. P. et al. Prognostic value of grip strength: findings from the Prospective Urban 
Rural Epidemiology (PURE) study. Lancet (London, England) 386, 266-73 (2015).

44. Celis-Morales, C. A. et al. Associations of grip strength with cardiovascular, respiratory, and cancer outcomes and all cause mortality: Prospective cohort study of half a million UK Biobank participants. BMJ 361, k1651 (2018).

45. Kamada, M., Shiroma, E. J., Buring, J. E., Miyachi, M. \& Lee, I.-M. Strength Training and All-Cause, Cardiovascular Disease, and Cancer Mortality in Older Women: A Cohort Study. $J$. Am. Heart Assoc. 6, (2017).

46. Stamatakis, E. et al. Does Strength-Promoting Exercise Confer Unique Health Benefits? A Pooled Analysis of Data on 11 Population Cohorts With All-Cause, Cancer, and Cardiovascular Mortality Endpoints. Am. J. Epidemiol. 187, 1102-1112 (2018).

47. UK Chief Medical Officers' physical activity guidelines. Available at: https://www.gov.uk/government/publications/physical-activity-guidelines-uk-chief-medicalofficers-report. (Accessed: 24th September 2019)

48. Ueda, P. et al. Laboratory-based and office-based risk scores and charts to predict 10-year risk of cardiovascular disease in 182 countries: a pooled analysis of prospective cohorts and health surveys. lancet. Diabetes Endocrinol. 5, 196-213 (2017). 